\title{
Applying Data Mining Techniques to Wafer Manufacturing
}

\author{
Elisa Bertino ${ }^{1}$, Barbara Catania ${ }^{2}$, and Eleonora Caglio ${ }^{3}$ \\ 1 Università degli Studi di Milano \\ Via Comelico 39/41 \\ 20135 Milano, Italy \\ bertino@dsi.unimi.it \\ 2 Università degli Studi di Genova \\ Via Dodecaneso 35 \\ 16146 Genova, Italy \\ catania@disi.unige.it \\ 3 Progres Progetti, \\ Via Varesina 76, \\ 20156 Milano, \\ dwh.dss@progres.it
}

\begin{abstract}
In this paper we report an experience from the use of data mining techniques in the area of semiconductor fabrication. The specific application we dealt with is the analysis of data concerning the wafer production process with the goal of determining possible causes for errors, resulting in lots of faulty wafers. Even though our application is very specific and deals with a specific manufacturing sector (e.g. semiconductor fabrication), we believe that our experience can be relevant to other manufacturing sectors and provide significant feedback on the use of data mining techniques.
\end{abstract}

\section{Introduction}

Background. In this paper we report an experience from the use of data mining techniques in the area of semiconductor fabrication. The problem we have considered refers to a specific request of a well known semiconductor producer. Such a request is related to the detection of the causes of failures, arising during the semiconductor manufacturing process. The semiconductor manufacturing process is a very complex activity composed of several phases (see [10] for details). The experience has shown that, if one of the constructed devices does not satisfy the design requirements, some problems arose during the so-called wafer fabrication phase.

The aim of the wafer fabrication step is that of creating several identical integrated circuits in and on the wafer surface. More precisely, given a wafer with a polished surface, during the wafer fabrication step a new wafer is generated whose surface contains several hundreds completed chips. In a single wafer fabrication phase, more than one hundred of individual steps are performed. However, each individual step is one of four basic phases, which are repeated several times. 
The phases are the following: layering, covering the wafer surface by a thin layer of conducting, semiconducting or insulating materials; pattering, removing some portions of the layers inserted in the layering phase, thus reproducing a specific pattern on the wafer surface; doping, putting specific amounts of dopants on the wafer surface, forming dopant regions and junctions that determine the electric characteristics of the semiconductor; heat treatments, during which the wafer is heated and cooled to achieve specific results.

Each main phase is then composed of several substeps, that may be repeated, each executed by a specific machine. The order by which such operations are executed depends on the type of the device that has to be constructed. In order to reduce time and costs, all the operations are often applied to wafer lots. A lot, that usually contains 25 wafers, is thus the processing unit.

The problem and the considered approach. In the considered application, during wafer fabrication, information about each step is collected in a production process database. This information, that refers to process data, electric misures, and so on, is used to establish the correctness of the produced devices. Whenever a wafer lot is faulty, production process engineers must go through the data collected in the production process database to find out possible causes for the production errors. This analysis is in general carried out "by hand", in the sense that all the tuples are extensively analyzed without the support of any automatic mechanism. Such an analysis may take quite a few days to the process engineers.

The goal of the experimental project we are describing was to use data mining techniques in order to reduce the analysis time from several days to a few hours. In our project, we used two commercially available data mining systems. One, MineSet, is a multistrategy system supporting several techniques [6]. The other system, Q-Yield, is, by contrast, specialized for manufacturing applications [5]. Both systems were not successful for our application and therefore we have developed our own analysis technique.

Related work. Because of the relevance of defect analysis in semiconductor manufacturing, issues related to management of defect-data has received some attention. In particular, a relevant effort is represented by the defect-data management system developed at SEMATECH [7], a non profit consortium of semiconductor manufacturers. However, no data mining tools are provided to help in determining possible explanations for the errors in the production. The use of data mining techniques for this specific problem is briefly discussed by Turney [8]. However, the emphasis of the discussion is on how to pre-process data in order to enable an effective use of a specific data mining technique. No specific experience is reported on the use of different techniques or systems.

Organization of the paper. The reminder of this paper is organized as follows. The data mining techniques and tools we have used in the project are described in Section 2. Section 3 presents the results we have obtained from the experiments. The new approach we propose is then introduced in Section 4. Finally, Section 5 presents some concluding remarks. 


\section{Data Mining Techniques and Systems Applied}

In the considered project, we were interested in two different types of data mining techniques: association rules and decision trees. Due to space constraints, we refer the reader to [4] for additional details about such techniques. Here we just recall their main characteristics.

Association rules. Let $I$ be a set of items. An association rule is a rule of the form $X \rightarrow Y$, where $X \subseteq I, Y \subseteq I, X \cap Y=\emptyset$. ${ }^{1}$ Such rule specifies that the presence of items $X$ determines the presence of items $Y$. An association rule $X \rightarrow Y$ has a support level $c$ in a set of tuples $T$ if the $c \%$ of tuples in $T$ satisfies $X$ and $Y$. It has a confidence level $c$ in $T$ if the $c \%$ of tuples in $T$ satisfying $X$ also satisfy $Y$. In general, it is useful to generate only the rules with a sufficiently high support.

Classification techniques. Classification is a function determining if an object belongs to a given class, chosen among a set of predefined classes, based on the values of some object attribute (the label). Among the proposed techniques, decision trees are becoming a largely used technique for classification [4]. The leaves of the tree correspond to all the possible values for the label. Each internal node is associated with an attribute and has a child for each value that can be associated with that attribute. Often, single values are replaced by mutually disjoint conditions. The disjunction of all the conditions appearing in the children of a given node completely characterizes the domain of the attribute. These properties guarantee that, given a certain item to be classified, there is always a single path from the root to a leaf whose conditions are satisfied by the item. Such path associates with the item the class corresponding to the reached leaf. Decision trees are typically constructed starting from a given set of tuples (the training set) and are then used to classify all the items.

In the context of the considered projects, two commercially available data mining systems, supporting the techniques described above, were used. One of them (MineSet) is a multistrategy system supporting several techniques, such as association rules, clustering techniques, and decision trees. The other system (Q-Yield) is, by contrast, specialized for manufacturing applications and uses a combination of Artificial Intelligence and statistics techniques to find relationships in production data. In the following, both systems are shortly described. ${ }^{2}$ MineSet. MineSet is a tool produced by Silicon Graphics [6]. The main data mining techniques provided by MineSet are the following:

- Association rules generator: the association rule generator produces a set of association rules, together with the support level and the confidence level.

- Classifiers: The tool supports two different types of classifiers, one based on decision-trees and one Naive-Bayes classifier [3]. In both cases, an attribute has to be identified as label; the classifier is constructed by considering a training set containing about $2 / 3$ of the considered data. Then, such classifier

\footnotetext{
${ }^{1}$ In a relational database, an item represents an association attribute/value.

${ }^{2}$ Both systems allow one to analyze both flat data and data contained in commercial relational DBMS.
} 
is used to classify additional data. For the aim of the project, we considered the decision tree classifier.

Q-Yield. Q-Yield is a single strategy tool produced by Quadrillion Corporation [5]. It is based on decision trees and it has been specifically designed to support designers in the detection of specific causes of a certain event and in making predictions. The tool provides the following main functionalities:

- It allows one to graphically represent how the values of a given attribute vary inside a relation. The graph may point out some exceptional situations (the event) that can be better analyzed by using the other tools provided by the system.

- The event can be described by declaratively specifying a logical condition on the considered data. Input data are then analyzed by the system to determine the causes (the logical conditions) of the specified event. The result is a set of rules, specifying the data conditions under which the event arises, together with some statistical information.

\section{Experiments and Results}

In order to address the problem described in Section 1, we have used both MineSet and Q-Yield on the production process database of a well known semiconductor producer. The process database is maintained in a relational database managed by the INGRES database management system (DBMS) [1]. A single relation is used to maintain all relevant information related to the production of a given device. Each tuple of such relation represents, among the others, the type of the operation performed by an equipment in a certain instant on a given wafer lot. The equipment (for example, the tube furnace used for layering) is identified by a string (eqt). The operation performed on a given wafer lot at a certain instant is identified by the triple (event,step,script), where event represents the main operation (for example, layering, patterning or doping), step identifies the specific step performed, and script provides additional information about the performed operation. The failure (the success) of an operation performed on a given wafer lot is represented by setting to 0 (1) the field fail in all tuples corresponding to that lot. Such setting is provided by process engineers during a pre-analysis step. During the production of a given product, the same operation can be executed on various equipments and the same step may correspond to various events. The process database typically contains data related to 80-90 lots, generated in a period of three-four months. Such data are very large in volume and are used for daily activities.

The goal of the experimental project we are describing was to use data mining techniques to detect the causes of process failure. A cause is certain if it allows one to determine all, and only those, lots that are faulty. A cause is uncertain if allows one to determine all lots that are faulty, together with some non-faulty lots. The problem we deal with is a typical problem for which Q-Yield should give good result since we exactly need to determine the causes of an event (generation 
of faulty wafers). In the following we present the results of the experiments we have carried out by using both tools.

\subsection{MineSet}

Association rules. The aim of the first group of experiments was to generate association rules representing the possible causes of faulty wafers. Therefore, the rules to be generated must have the form: attribute $_{1}=$ value $_{1} \wedge \ldots \wedge$ attribute $_{n}=$ value $_{n} \rightarrow$ fail $=0$. The experiments we have carried out are based on the following choices:

- We have projected the starting relation on different sets of attributes. Indeed, the result of the application of data mining techniques strongly depends on the input set of data. By changing the starting set, different results are usually obtained.

- MineSet allows one to specify the required confidence and support levels. We have considered two main confidence levels:

(a) Confidence $=50 \%$ : with this setting, we restrict the system to generate significant rules. However, since the confidence is $50 \%$, the generated rules does not necessarily return certain causes.

(b) Confidence $=100 \%$ : with this setting, only certain causes are generated by the system.

Since the number of faulty lots is typically low with respect to the total number of lots, the support of association rules describing the causes of failure should be low. For this reason, we have set the support level to $1 \%$ and to $0.5 \%$.

The obtained results have not been very satisfactory. By setting the support level to $0.5 \%$, no rules have been generated. By setting the support level to $1 \%$, in case (a), the tool has generated some rules, similar to the following ones:

$$
\begin{array}{ccc}
\text { Support } & \text { Confidence } & \text { Rules } \\
1.3096 & 92.06 & \text { eqt }=P E 06 \rightarrow \text { fail }=1 \\
1.4902 & 89.19 & \text { eqt }=\text { WJ } 1 \rightarrow \text { fail }=1
\end{array}
$$

Such rules do not characterize faulty lots of wafers but correct lots. No rule with fail $=0$ on the right side has been generated. In case (b), no rule has been generated. By deeply analyzing the tool, we have discovered that this behavior depends on the fact that MineSet generates only rules having a support greater than $1 \%$ and a confidence higher than $50 \%$. This assumption makes MineSet association rules useless in determining causes of failure in our application.

Decision trees. The aim of the second group of experiments was to extract information about faulty lots by using decision trees. We have performed several experiments by varying the set of attributes of the starting relation and by varying the label field. In all the experiments, the resulting decision trees represent only non significant information. For example, if we choose fail as label, we 
obtain a 1-level tree, in which each leaf corresponds to a specific value for the wafer lot. No causes are determined, since no attributes, besides lot and fail, are considered. Similar results have been obtained by varying the starting set of attributes.

Starting from these results, we have analyzed in depth the algorithm for the construction of decision trees and we have discovered that the maximum number of children for a node is 25 . In our case, the only attribute with less than 25 values is lot. Therefore, for our application, we need decision trees able to support a much larger number of children for a given node. Moreover, we have observed that decision trees generate significant results only when attribute values can be significantly compared. This happens when an attribute takes only few different values in the input relation and when such values are numbers. In our case, both conditions are not satisfied and this is the reason why such technique fails. As an additional experiment, we have transformed values for eqt in numerical values, choosing lot as label. The obtained tree allows one to determine with a certain confidence, some numerical intervals containing the equipments processing some faulty lots of wafer. However, the conditions under which all faulty wafer lots have been generated have not been determined.

\subsection{Q-Yield}

In order to use Q-Yield, a condition representing the event to be analyzed has to be specified. In our case, the condition is $f a i l=0$. Each rule returned by the system in the performed experiments specifies some conditions on equipments and on operations under which the event is satisfied. The following are some examples of rules returned by the system:

$\begin{array}{lcccc}\text { Rule } & \text { Coverage } & \text { Error rate Correct } & \text { Incorrect } \\ \text { eqt }=S T Z Z 1 & 1.0 \% & 0.0 \% & 8 & 0 \\ e q t \neq S T K Z 1 \wedge e q t \neq S T K P 4 & 0.5 \% & 0.0 \% & 4 & 0 \\ \quad \wedge e q t=16870 & & & & \\ \text { eqt } \neq S T K Z 1 \wedge e q t=S T K P 4 & 1.0 \% & 27.3 \% & 8 & 3\end{array}$

In the previous rules, error rate (incorrect) represents the percentage (the number) of tuples satisfying the rule but not the event whereas coverage (correct) represents the percentage (the number) of tuples satisfying the rule and the event. From the previous rules, if we know the number of faulty lots and if the number of tuples is equal to the number of lots, the exact causes of the failures are represented by the rules for which the number of tuples making the rule correct coincides with the number of faulty lots. However, in our application, each lot appears in several tuples. Thus, the previous method cannot be applied. The only way to get this answer is to consider the rules satisfied by a number of tuples which is higher or equal to the number of faulty lots (representing uncertain causes) and then directly analyze the file. Since the number of tuples is very high, this solution does not seem reasonable.

Even if also Q-Yield was not able to determine certain causes, it returned more information than MineSet tools. An additional advantage of Q-Yield with 
respect to MineSet is that it works well on both numerical and non-numerical attributes. This is the reason why we get some results.

\section{A New Approach}

The analysis of the performed experiments shows that current data mining techniques are not adequate to solve the considered problem. Such inadequacy is mainly due to two different reasons: (i) the considered problem is related to the detection of the causes of a non frequent event: in general, data mining tools are successfully used when some regularities in the data set has to be found; (ii) even if the data considered in the experiments are exactly the data that the semiconductor producer daily uses in its activity, they are not significant from the point of view of data mining techniques: each attribute contains values that are not easily comparable and the set of active values is typically very high.

The considered problem is therefore a typical case in which data mining techniques may fail. Therefore, we have designed an ad hoc algorithm. Though this algorithm has been designed for fault analysis in the semiconductor manufacturing process, it can also be used in other manufacturing processes characterized by the following properties:

- The process database is composed of a single relation, called process relation, containing non necessarily numerical attributes. Each tuple in the relation contains information about a given process step.

- With each tuple, an information is associated concerning the result of the process operation (success/failure) (attribute fail). Success/failure is assumed to be related to a specific element (a lot, in our application).

- The aim of the analysis is the detection of the certain or uncertain causes of failure.

In the following, we first present the general algorithm, then we describe the obtained results (see [2] for additional details).

\subsection{Definition of the Approach}

Given a manufacturing process, characterized by the properties described above, the causes that have to be detected are generally represented by specific assignments of values to a subset of the attributes belonging to the problem domain. For example, in the domain under consideration, the engineers can be interested in determining the equipments always generating failures, the operations always generating failures or the time intervals at which all failing lots have been generated. If no equipment, no operation, no time instant represent a certain cause, a combination of them may correspond to the solution, identifying, for example, that an operation performed on a given instant of time on a certain equipment was the cause of the problem.

Starting from the previous considerations, in order to determine certain and uncertain causes of a given event, it is important to specify which attribute combinations have to be considered. The combinations are assumed to be partially 
ordered. Such ordering specifies which combination has to be considered first and how combinations can be refined. After that, we have to determine which attribute assignments represent certain or uncertain causes. In the following, both steps are analized in more detail.

Representation of significant combinations. Let $D$ be the set of attributes of the process relation. Let $S_{1}, \ldots, S_{n} \subseteq 2^{D}$ be all significant combinations, as specified by process engineers. Let $\preceq$ be a partial order on $\left\{S_{1}, \ldots, S_{n}\right\}$, called interest order, such that if $S_{i} \preceq S_{j}$, the engineers are first interested in determining if $S_{i}$ is a certain cause. If it is not, but it is an uncertain cause, they are interested in refining $S_{i}$ in $S_{j}$, to determine if $S_{j}$ is a certain cause. Significant combinations and the interest order can be represented as a direct graph, called interest graph, having the significant combinations as nodes and one edge $\left(S_{i}, S_{j}\right)$ if and only if $S_{i} \preceq S_{j}$. All the nodes in the graph having an empty fan-in represent all combinations from which the analysis has to start.

Causes detection. Let $G$ be an interest graph. If an edge $\left(S_{i}, S_{j}\right)$ exists in $G$, $S_{i}$ has to be analyzed before $S_{j}$ and $S_{j}$ has to be analyzed only if no assignment for $S_{i}$ represents a certain cause. This visit corresponds to a slightly modified breadth first search of the graph. In particular, the search from a node must stop if no certain or uncertain causes can be associated with that node. In the following, we assume that two functions, certain and uncertain, exist, taking a significant combination and returning the set of assignments representing certain or uncertain causes, respectively.

In order to correctly modify the breadth first search, we associate with each node an additional attribute marked, initialized to true. Let $n$ be a node visited by a breadth first search associated with a significant combination $S$. If mar$\operatorname{ked}(n)=$ true, we determine if there exists an assignment for $S$ representing a certain cause $(\operatorname{certain}(S) \neq \emptyset)$. If it exists, we return $\operatorname{certain}(S)$ to the user and we set $\operatorname{marked}(n)$ to false. If it does not exist, we determine if there exists an assignments for $S$ representing an uncertain cause (uncertain $(S) \neq \emptyset$ ). If it does not exist, we set marked( $n$ ) to false. When all nodes have been visited, if no certain cause has been returned to the user, we report the uncertain causes. Such causes are generated from the combinations associated with all nodes $n$ having $\operatorname{marked}(n)=$ true.

In order to complete the description of the algorithm, we have to specify how functions certain and uncertain are implemented. Since we assume that the process relation is stored in a relational DBMS, simple SQL aggregated queries can be used to determine certain and uncertain causes. Due to space constraints, we refer the reader to [2] for additional details.

A tool has been implemented based on the previous algorithm, under the database management system Informix, using ESQL/C as programming language. 


\subsection{Results}

The attributes that are typically used in the analysis are lot, eqt, event, step, time, and fail (see Section 3). ${ }^{3}$ From the requirement analysis, we have discovered that the interest graph of the considered application is indeed a tree and has the form presented in Figure 2. This means that process engineers are first interested in finding the equipments generating failures. In this case, an hardware problem is detected: an equipment does not work as it should do. If no equipment represents a certain cause for the problem, the event is also considered. If neither in this case a certain cause is detected, the step is taken into account. In both situations, a problem still concerning an equipment is detected. However, in this case, it refers a specific equipment functionality. As a final refinement, the time is considered. Different time aggregations, with different granularities (day, week), have been considered. In this case, the cause could represent an accidental problem happened at a certain time. Note that in the considered application, a monotonic refinement of combinations is used: a combination is always refined by adding attributes.

The algorithm presented in Subsection 4.1 has then be applied to the same datasets on which we have applied data mining techniques. In most cases, the proposed algorithm determined the certain causes, often represented by a single equipment (identifying an hardware problem). In some other cases, it determined only uncertain causes, often represented by some assignments for (eqt, event, step). This information has then been used by the process engineers to further investigate the problem. They discovered that, when no certain causes are determined, the faults are mainly due to some accidental problem.

\section{Concluding Remarks}

This paper has reported the results we have obtained in investigating the effectiveness of current data mining techniques in determining the causes of failures of a wafer fabrication process. The experiments we carried out have shown that data mining techniques fail to solve this problem. We have therefore proposed a new approach tailored to the detection of the causes of failure in manufacturing processes.

As overall conclusion, we would like to point out that, in addition to technical problems that we found out in our experiments (see Section 3), we noticed some more general problems in applying data mining techniques to the semiconductor manufacturing process. The first problem is that it is difficult to determine right away which specific data mining technique to use for the problem at hand. Another problem is that data must often be pre-processed before being used for data mining. Such pre-processing can be non trivial and depends on the specific data mining technique one expects to use. Both problems point out the need of some general methodologies and guidelines that could support the users in the development of data mining applications.

\footnotetext{
3 In the requirement analysis for the considered application, we have noted that often attribute script is useless since it often contains redundant information.
} 


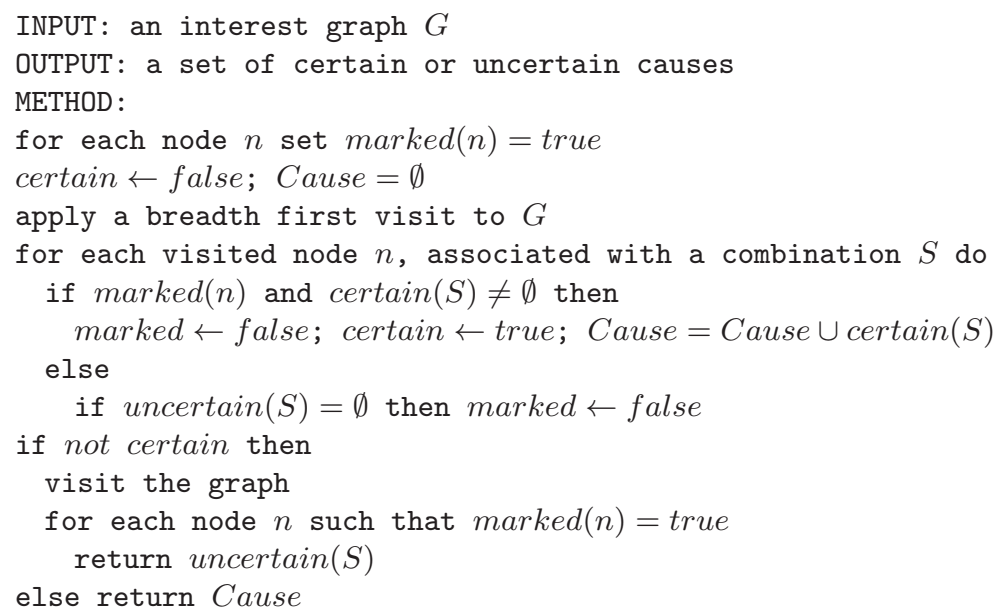

Fig. 1. The faults detection algorithm

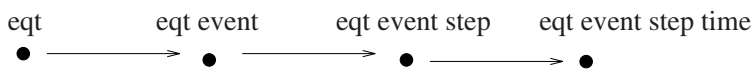

Fig. 2. The interest graph corresponding to the semiconductor manufacturing process

\section{References}

1. ASK Computer Co: INGRES/SQL Reference Manual.

2. Bertino, E., Catania, B.: Applying Data Mining Techniques to Wafer Manufacturing. Technical Report, University of Milano, Italy (1999)

3. Cheeseman, P., Stutz, J.: Bayesian Classification (Autoclass): Theory and Results. In Fayyad, U.M., Piatetsky-Shapiro, G., Smith, P., Uthurusamy, R. (eds): Advances in Knowledge Discovery and Data Mining. AAAI/MIT Press (1996)

4. Fayyad, U.M., Piatetsky-Shapiro, G., Smith, P., Uthurusamy, R.: Advances in Knowledge Discovery and Data Mining. AAAI/MIT Press (1996)

5. Q-Yield Product Information. http://www.quadrillion.com/qyover.htm

6. Silicon Graphics MineSet - Supporting the Discovery Research Process. Silicon Graphics White Paper, http://www.sgi.com/ software/mineset/mineset_data.html (1997)

7. Singh, H., Lakhani, F., Proctor, P., Kazakoff, A.: Defect Data Management System at SEMATECH. Solid State Technology 75-80. Pennwell Publishing (1995)

8. Turney, P.: Data Engineering for the Analysis of Semiconductor Manufacturing Data. In Proc. of the IJCAI-95 Workshop on Data Engineering for Inductive Learning (1995)

9. Viveros, M.S., Nearhos, J.P., Rothman, M.: Applying Data Mining Techniques to a Health Insurance Information System. In Proc. the 22nd International Conference on Very Large Databases (1996)

10. van Zant, P.: Microchip Fabrication. McGraw-Hill (1997) 